\title{
HRCT features between lepidic- predominant type and other pathological subtypes in early-stage invasive pulmonary adenocarcinoma appearing as a ground- glass nodule
}

Pengju Zhang ${ }^{1+}$, Tianran $\mathrm{Li}^{1+}$, Xuemin Tao ${ }^{2}$, Xin $\mathrm{Jin}^{2}$ and Shaohong Zhao ${ }^{2^{*}}$

\begin{abstract}
Background: Different pathological subtypes of invasive pulmonary adenocarcinoma (IPA) have different surgical methods and heterogeneous prognosis. It is essential to clarify IPA subtypes before operation and high-resolution computed tomography (HRCT) plays a very important role in this regard. We aimed to investigate the HRCT features of lepidic-predominant type and other pathological subtypes of early-stage (T1NOM0) IPA appearing as a ground-glass nodule (GGN).

Methods: We performed a retrospective analysis on clinical data and HRCT features of 630 lesions in 589 patients with pathologically confirmed IPA (invasive foci $>5 \mathrm{~mm}$ ) appearing as pure GGN (pGGN) and mixed GGN (mGGN) with consolidation-to-tumor ratio (CTR) $\leq 0.5$ from January to December 2019. All GGNs were classified as lepidicpredominant adenocarcinoma (LPA) and nonlepidic-predominant adenocarcinoma (n-LPA) groups. Univariate analysis was performed to analyze the differences of clinical data and HRCT features between the LPA and n-LPA groups. Multivariate analysis was conducted to determine the variables to distinguish the LPA from n-LPA group independently. The diagnostic performance of different parameters was compared using receiver operating characteristic curves.

Results: In total, 367 GGNs in the LPA group and 263 GGNs in the n-LPA group were identified. In the univariate analysis, the CTR, mean CT values, and mean diameters as well as mixed GGN, deep lobulation, spiculation, vascular change, bronchial change, and tumor-lung interface were smaller in the LPA group than in the n-LPA group $(P<0.05)$. Logistic regression model was reconstructed including the mean $C T$ value, $C T R$, deep lobulation, spiculation, vascular change, and bronchial change $(P<0.05)$. Area under the curve of the logistic regression model for differentiating LPA and n-LPA was 0.840 (76.4\% sensitivity, 78.7\% specificity), which was significantly higher than that of the mean CT value or CTR.
\end{abstract}

\footnotetext{
* Correspondence: zhaoshaohong@aliyun.com

${ }^{\dagger}$ Pengju Zhang and Tianran Li contributed equally to this work.

${ }^{2}$ Department of Radiology, First Medical Center of PLA General Hospital, 28 Fuxing Road, Beijing 100853, China

Full list of author information is available at the end of the article
}

C C The Author(s). 2021 Open Access This article is licensed under a Creative Commons Attribution 4.0 International License, which permits use, sharing, adaptation, distribution and reproduction in any medium or format, as long as you give appropriate credit to the original author(s) and the source, provide a link to the Creative Commons licence, and indicate if changes were made. The images or other third party material in this article are included in the article's Creative Commons. licence, unless indicated otherwise in a credit line to the material. If material is not included in the article's Creative Commons licence and your intended use is not permitted by statutory regulation or exceeds the permitted use, you will need to obtain permission directly from the copyright holder. To view a copy of this licence, visit http://creativecommons.org/licenses/by/4.0/ The Creative Commons Public Domain Dedication waiver (http://creativecommons.org/publicdomain/zero/1.0/) applies to the data made available in this article, unless otherwise stated in a credit line to the data. 
Conclusions: Deep lobulation, spiculation, vascular change, and bronchial change, CT value $>-472.5 \mathrm{HU}$ and CTR $>27.4 \%$ may indicate nonlepidic predominant invasive pulmonary adenocarcinoma in GGNs.

Keywords: Lung neoplasms, Ground glass nodule, Tomography, X-ray computed, Pathology

\section{Background}

With the development and popularization of highresolution computed tomography (HRCT) lung cancer screening, the detection rate of ground-glass nodules (GGNs) has significantly increased [1, 2]. Numerous studies have confirmed that most long-term existed GGNs in the lung are mostly early lung adenocarcinoma or their precancerous lesions [3]. According to whether or not it contained a solid component, a GGN can be classified as pure GGN (pGGN) and mixed GGN (mGGN), a mGGN is divided into ground glasspredominant mGGN $(0<\mathrm{CTR} \leq 0.5)$ and solidpredominant mGGN $(0.5<\mathrm{CTR}<1)$ according to a consolidation-to-tumor ratio (CTR) on HRCT [4]. The 2015 World Health Organization (WHO) Classification of Lung Tumors [5] classified invasive pulmonary adenocarcinoma (IPA) into lepidic-predominant adenocarcinoma (LPA), acinar-predominant adenocarcinoma (APA), papillary-predominant adenocarcinoma (PPA), micropapillary-predominant adenocarcinoma (MPA), solid-predominant adenocarcinoma (SPA) according to the main growth patterns. Among them, LPA has the best prognosis, followed by the APA and PPA, whereas SPA and MPA have the worst [6, 7]. Different pathological subtypes of early-stage IPA may have different surgical methods, LPA may be treated with partial lobectomy (segmentectomy or wedge resection) and no lymph node dissection, whereas other subtypes can be treated with standard therapy for anatomical lobectomy and systemic lymph node dissection [7-9]. Preoperative puncture pathology and intraoperative freezing pathology are helpful for the diagnosis of pathological subtypes of lung adenocarcinoma, but both are limited by materials, and the risk of lung adenocarcinoma subtypes is underestimated [10]. HRCT can reflect the overall aggressiveness of nodules, and can help to improve the accuracy of pathological subtype diagnosis. Previous studies [11-13] have shown that HRCT features of GGN have high diagnostic value in differentiating early IPA from preinvasive lesions (PIL) and minimally invasive adenocarcinoma (MIA), but there are few studies on the correlation of HRCT features among subtypes of IPAs. Therefore, it is essential that HRCT features should be used to identify LPA from other subtypes in IPAs with GGN.

In this study, the HRCT features of IPAs appearing as pGGN and mGGN $(C T R \leq 0.5)$ were retrospectively analyzed to determine imaging differences between the LPA and other pathological subtypes to provide imaging help for clinical surgical decision-making.

\section{Methods}

This study was approved by the Ethics Committee of Fourth Medical Center of Chinese PLA General Hospital (approval No. 2019YL002-HS001). All participants and/ or their family members provided the informed consent.

\section{Participants}

The clinical and CT imaging data of patients having a confirmed diagnosis of IPA based on pathological results and undergoing resection at the Department of Thoracic Surgery of our hospital from January to December 2019 were collected. The inclusion criteria were as follows: (1) early-stage (T1NOM0) lung adenocarcinoma was confirmed by surgical pathology; (2) preoperative CT images appearing as GGN; (3) no chemoradiotherapy, no needle biopsy and no endoscopy were performed before the CT examination. The exclusion criteria were as follows: (1) PIL, MIA and variants of IPA; (2) no routine CT examination or $1 \mathrm{~mm}-1.25 \mathrm{~mm}$ thin-slice imaging performed within 1 month preoperatively; (3) heavy respiratory artifacts, or GGNs with air space type affecting the measurement of the CT value; (4) GGNs with a maximum diameter $>3 \mathrm{~cm}$ or CTR $>0.5$ on CT. Finally, $589 \mathrm{pa}-$ tients (630 GGNs in total) were included for analysis (Fig. 1). Multiple GGNs in one patient were analyzed as independent lesions.

A total of 630 IPAs with invasive foci $>5 \mathrm{~mm}$ were divided into lepidic-predominant adenocarcinoma (LPA) and nonlepidic-predominant adenocarcinoma (n-LPA) groups. The subtypes of all IPAs in this study were recorded according to the pathology reports. The LPA group only included LPA subtype, whereas the n-LPA group included APA, PPA, MPA, and SPA subtypes. As a small number of micropapillary structures in IPA were strongly associated with poor prognosis [5], IPA with micropapillary structure was classified as n-LPA group in our study. One radiologist screened the participants and recorded the relevant clinical data.

\section{Inspection method}

Brilliance iCT256 CT scanner (Philips Medical Systems, Netherland) or GE Optima 64 spiral CT scanner (GE Healthcare Technologies, Waukesha, WI) was used for conventional chest CT volume scanning. The scanning range was from the lung apex to the posterior 
2067 patients with surgical removal of pulmonary adenocarcinoma nodules in the Department of Thoracic Surgery of our hospital from January to December 2019

302 PIL-MIAs and 6 invasive mucinous adenocarcinomas pathologically were exclued

No conventional CT examination or HRCT images in 114 patients within one month before surgery

Respiratory motion artifacts in $10 \mathrm{GGNs}$, and nine GGNs presented with air space, and its $\mathrm{CT}$ value could not be accurately measured.

On HRCT, the maximum diameter $>3 \mathrm{~cm}$ of 179 GGNs or the CTR $>0.5$ of $283 \mathrm{GGNs}$

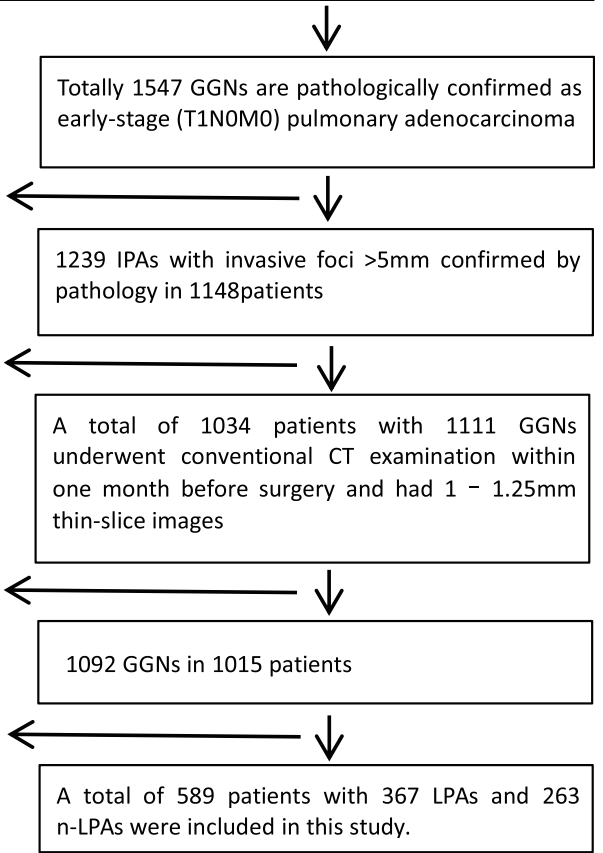

Fig. 1 Flow chart of patient screening. HRCT, high-resolution computed tomography; IPA, invasive pulmonary adenocarcinoma; GGN, groundglass nodule; PIL-MIA, preinvasive lesions-minimally invasive adenocarcinoma; CTR, consolidation-to-tumor ratio; LPA, lepidic-predominant adenocarcinoma; n-LPA, nonlepidic-predominant adenocarcinoma

costophrenic angle. The CT scan parameters were as follows: tube voltage, $120 \mathrm{KV}$; pitch, 0.993, 1.375; matrix, $1024 \times 1024,512 \times 512$; scanning slice thickness, $5 \mathrm{~mm}$; reconstruction slice thickness, $1.25 \mathrm{~mm}$ or $1 \mathrm{~mm}$; use of sharp and standard algorithms; lung window (window width, $1500 \mathrm{HU}$ and window level, $-600 \mathrm{HU}$ ); and mediastinal window (window width, $400 \mathrm{HU}$ and window level, $40 \mathrm{HU})$.

\section{Pathologic evaluation}

The surgically resected specimens were fixed in 10\% formalin, embedded in paraffin, sliced with a microtome, and stained with HE. All specimens were classified according to the criteria of the 2015 WHO Classification of Lung Tumors [5]. These tumors should be classified according to the main growth mode of the tumor and semiquantitatively evaluated the proportion of various growth patterns in 5\% increments. The percentage of each growth pattern was recorded in the report. All included cases were reconfirmed by an experienced pulmonary pathologist.

\section{Analysis of clinical data and HRCT features}

Clinical data were collected from the thoracic surgery database of our hospital, including patient's age, sex, smoking history, and surgical records.
The HRCT features, including continuous and categorical variables were browsed and analyzed on the Picture Archiving \& Communication System. Several categorical variables included location, nodule type, deep lobulation, spiculation, vascular change, bronchial change, bubble-like lucency, pleural indentation, tumor-lung interface. The nodule type is pGGN and mGGN. Deep lobulation is characterized as a scalloplike lobulation on the nodule surface, and the maximum single lobulation chord distance/chord length is $\geq 0.4$ (Fig. 2). Vascular change refers to the dilation, stiffness, distortion, and aggregation of blood vessels. Bronchial change refers to dilation, distortion, and truncation. The tumor-lung interface is a clear or fuzzy boundary between the tumor and adjacent normal lung tissue.

Three continuous variables were record. The first variable was the mean diameter, which refers to the mean value of the longest diameter $\left(D_{\max }\right)$ of the nodule measured at the maximum level of the lung window and the maximum diameter $\left(D_{\mathrm{per}}\right)$ perpendicular to it (Fig. 3). The calculation method is as follows: $\left(D_{\max }+D_{\text {per }}\right) / 2$. The second was CTR which is calculated by $S_{\max } / D_{\max } \times 100 \%, S_{\max }$ is the maximum diameter of solid component measured by adjusting window level and window width (WL, $-160 \mathrm{HU}$; WD, 


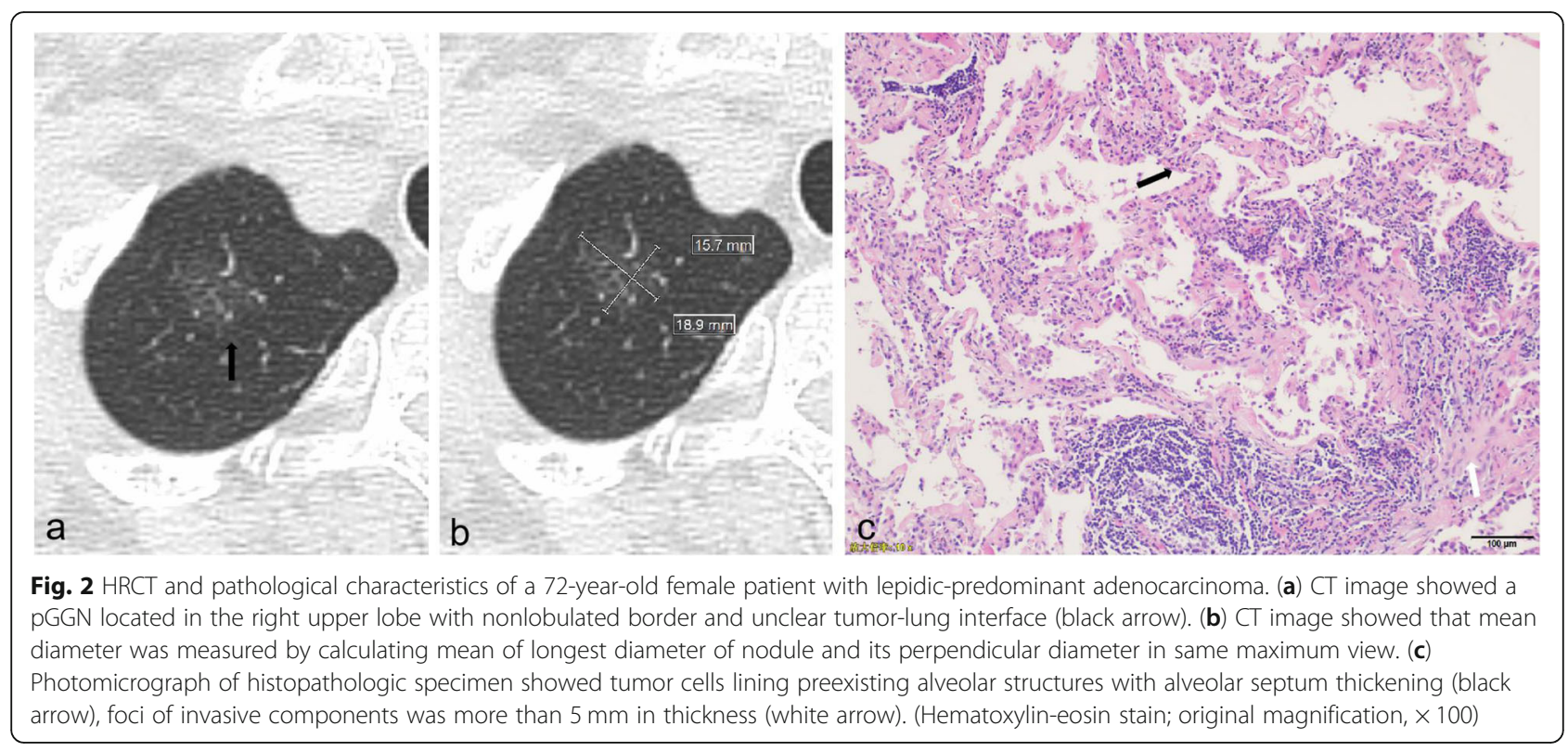

2HU) [14] (Fig. 4). The last was the mean CT value which is measured by the Freehand region of interest (ROI) along the edge of the nodule on the maximum axis of GGN and upper and lower adjacent slices on the lung window, avoiding the bronchioles, blood vessels, and air-containing cysts as much as possible, and taking the mean value of three measurements as the mean $\mathrm{CT}$ value.

Two chest radiologists with $>15$ years of working experience independently analyzed the images without knowing the clinical data and pathological diagnosis of the patients. Any inconsistent results were solved after discussion.

\section{Statistical analysis}

When comparing the LPA and n-LPA groups, two independent sample $t$ tests were used to assess continuous variables conforming to normal distribution, while Mann-Whitney $U$ tests were used to compare nonnormally distributed data. Continuous variables included age, mean diameter, mean CT value, and CTR. Categorical variables were analyzed using the Pearson chi-square test and Fisher's exact test, including sex, smoking history, and HRCT features. The binary logistic regression analysis was performed on continuous and categorical variables with statistical significance in the univariate analysis. A simple logistic regression model was created

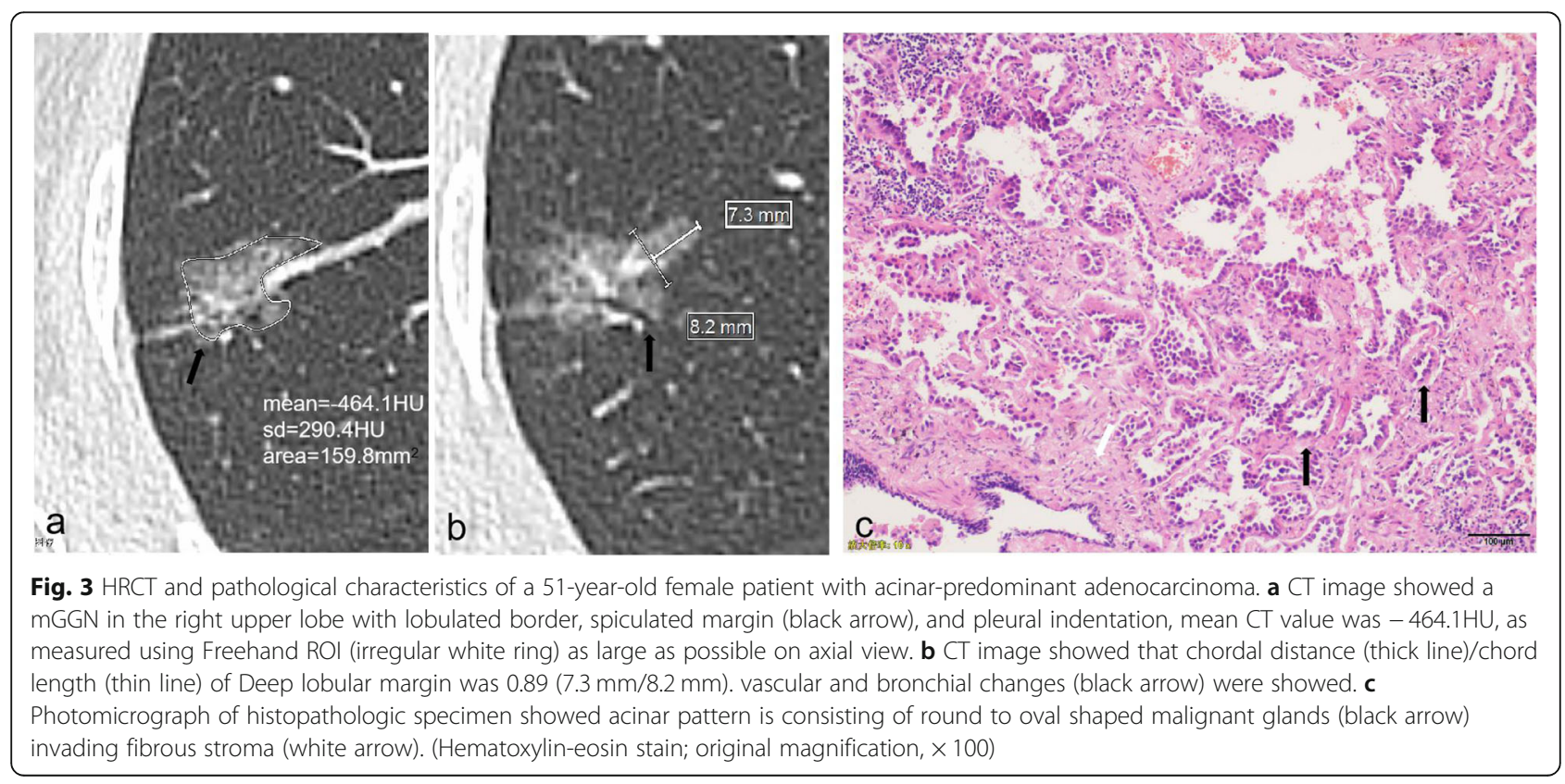



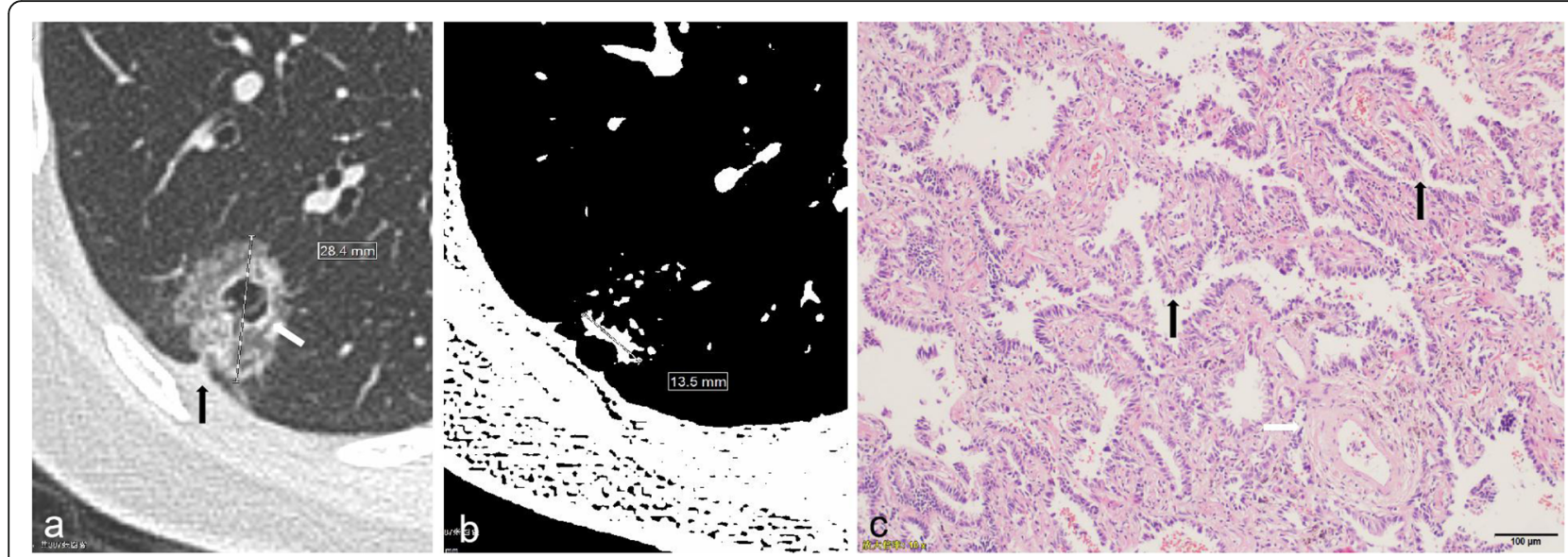

Fig. $4 \mathrm{HRCT}$ and pathological characteristics of a 55-year-old female patient with papillary-predominant adenocarcinoma. a CT image showed mGGN in the right lower lobe with spiculation, pleural indentation (black arrow) and bubble-like lucency (white arrow). The maximum diameter of GGN (Dmax) was measured. $\mathbf{b}$ CT image showed that the maximum diameter of solid component (Smax) was measured by adjusting window level and the window width (WL: -160HU; WD: 2HU). CTR=47.5\% (Smax/Dmax; $13.5 \mathrm{~mm} / 28.4 \mathrm{~mm}$ ). c Photomicrograph of histopathologic specimen showed a papillary pattern (black arrow) with cuboidal tumor cells growing along the surface of fibrovascular cores. Pulmonary vein was seen (white arrow). (Hematoxylin-eosin stain; original magnification, $\times 100)$

using the backward elimination process. The receiver operating characteristic (ROC) analysis was performed using the logistic regression model and continuous variables with statistical significance in the multivariate analysis. The area under the curve (AUC) was used to evaluate the discrimination efficiency of the model in the LPA and n-LPA groups. The cutoff value was defined as the maximum value of the Youden's index. All univariable and multivariate analyses were performed using IBM SPSS version 21.0 software. A $P$-value of < 0.05 was considered statistically significant.

\section{Results}

The clinical findings of the patients were summarized in Table 1. Among 589 patients, 203 were men and 386 were women; the average age was 55 years (range, 2378 years); 122 were smokers, 462 were nonsmokers, and five had an unknown smoking history. Of these patients, 260 patients underwent one-lobe lobectomy, 269 underwent single-wedge resection or segmental resection, one underwent two-lobe lobectomy (right upper lobe + middle lobe), 25 underwent one-lobe lobectomy combined with one-segmental or wedge resections, and 31 with two lesions and three with three lesions underwent multiple segmental or wedge resections. Of all GGNs, one GGN was detected in 553 patients, two GGNs in 32 patients, three in 3 patients and four in 1 patient.

Among 630 GGNs, 367 GGNs were in the LPA and 263 in the n-LPA groups including 221 APAs, 28 PPAs, three SPAs, and 11 IPAs with micropapillary structure. No significant differences were found in sex, age, and smoking history between the LPA and n-LPA groups $(P=0.522,0.663$, and 0.396 , respectively).

Table 1 Clinical data analysis of GGN

\begin{tabular}{|c|c|c|c|c|}
\hline \multirow[t]{2}{*}{ Clinical information } & \multirow{2}{*}{$\begin{array}{l}\text { Total } \\
\text { number of } \\
\text { patients } \\
(n=589)\end{array}$} & \multicolumn{2}{|c|}{ Number of GGNs $(n=630)$} & \multirow[t]{2}{*}{$P$} \\
\hline & & LPA(367) & n-LPA(263) & \\
\hline Age (year), mean \pm standard deviation & $55.03 \pm 10.07$ & $54.91 \pm 9.82$ & $55.26 \pm 10.19$ & 0.663 \\
\hline Sex & & & & 0.522 \\
\hline Male & 203 & 129 & 86 & \\
\hline Female & 386 & 238 & 177 & \\
\hline Smoking history (5 missing cases) & & & & 0.396 \\
\hline No & 462 & 293 & 205 & \\
\hline Yes & 122 & 70 & 58 & \\
\hline
\end{tabular}

GGN Ground-glass nodule, LPA Lepidic-predominant adenocarcinoma, $n$-LPA Nonlepidic-predominant adenocarcinoma. $P<0.05$ was considered statistically significant 
Table 2 High-resolution computed tomography features of ground-glass nodules

\begin{tabular}{|c|c|c|c|c|}
\hline Features & Classification & LPA (367) & n-LPA (263) & $P$ \\
\hline \multicolumn{2}{|c|}{ Mean diameter $(\mathrm{mm})$, mean \pm standard deviation } & $14.49 \pm 5.22$ & $15.43 \pm 4.79$ & 0.021 \\
\hline \multicolumn{2}{|c|}{ Mean $C T$ value $(H U)$, mean \pm standard deviation } & $-566.1 \pm 98.2$ & $-449.3 \pm 111.5$ & $<0.001$ \\
\hline \multicolumn{2}{|l|}{$\mathrm{CTR}(\%), M(Q 1, Q 3)$} & $13.9(0,27.3)$ & $27.8(7.7,40)$ & $<0.001$ \\
\hline \multirow[t]{5}{*}{ Location } & RUL & 146 & 95 & \multirow[t]{5}{*}{0.477} \\
\hline & RML & 29 & 17 & \\
\hline & RLL & 52 & 45 & \\
\hline & LUL & 95 & 64 & \\
\hline & LLL & 45 & 42 & \\
\hline \multirow[t]{2}{*}{ Type } & $\mathrm{pGGN}$ & 262 & 149 & \multirow[t]{2}{*}{$<0.001$} \\
\hline & mGGN & 105 & 114 & \\
\hline \multirow[t]{2}{*}{ Deep lobulation } & No & 148 & 35 & \multirow[t]{2}{*}{$<0.001$} \\
\hline & Yes & 219 & 228 & \\
\hline \multirow[t]{2}{*}{ Spiculation } & No & 280 & 101 & \multirow[t]{2}{*}{$<0.001$} \\
\hline & Yes & 87 & 162 & \\
\hline \multirow[t]{2}{*}{ Vascular change } & No & 206 & 53 & \multirow[t]{2}{*}{$<0.001$} \\
\hline & Yes & 161 & 210 & \\
\hline \multirow[t]{2}{*}{ Bronchial change } & No & 92 & 40 & \multirow[t]{2}{*}{0.003} \\
\hline & Yes & 275 & 223 & \\
\hline \multirow[t]{2}{*}{ Bubble-like lucency } & No & 314 & 218 & \multirow[t]{2}{*}{0.374} \\
\hline & Yes & 53 & 45 & \\
\hline \multirow[t]{2}{*}{ Pleural indentation sign } & No & 178 & 120 & \multirow[t]{2}{*}{0.518} \\
\hline & Yes & 189 & 143 & \\
\hline \multirow[t]{2}{*}{ Tumor-lung interface } & Unclear & 122 & 46 & \multirow[t]{2}{*}{$<0.001$} \\
\hline & Clear & 245 & 217 & \\
\hline
\end{tabular}

LPA Lepidic-predominant adenocarcinoma, $n$-LPA Nonlepidic-predominant adenocarcinoma, CTR Consolidation-to-tumor ratio, $p G G N$ Pure ground-glass nodule, $m G G N$ Mixed ground-glass nodule, RUL Right upper lobe, $R M L$ Right middle lobe, $R L L$ Right lower lobe, LUL Left upper lobe, LLL Left lower lobe. $P<0.05$ was considered statistically significant

\section{Analyzing HRCT features of GGNs}

The mean diameter, mean CT value, and CTR were significantly smaller in the LPA group than in the n-LPA group (14.49 $\mathrm{mm}$ vs. $15.43 \mathrm{~mm}, P=0.021$; $-566.1 \mathrm{HU}$ vs. $-449.3 \mathrm{HU}, P<0.001 ; 13.9 \%$ vs. $27.8 \%, P<0.001)$. The location of GGN was as follows: 241 in the right upper lobe, 46 in the right middle lobe, 97 in the right lower lobe, 159 in the left upper lobe, and 87 in the left lower lobe. No significant difference was observed in the location, bubble-like lucency, and pleural indentation sign between the LPA and n-LPA groups $(P=0.477,0.374$, and 0.518 , respectively). The $\mathrm{mGGN}$, deep lobulation, spiculation, vascular change, bronchial change, and clear tumorlung interface were more common in the n-LPA group than LPA group $(P<0.001,<0.001,<0.001,<0.001,=$ 0003, and $<0.001$, respectively) (Table 2 ).

\section{Logistic regression analysis and ROC analysis of GGNs}

Binary logistic regression analysis was performed on HRCT features with statistical significance in univariate analysis, including mean diameter, mean CT value, CTR, deep lobulation, spiculation, vascular change, bronchial change, and tumor-lung interface. As nodule type was strongly associated with CTR, nodule type was excluded from logistic regression analysis. Binary logistic regression analysis showed that high $\mathrm{CT}$ value $[P<0.001$; odds ratio $(\mathrm{OR})=1.009]$, deep lobulation $(P<0.001$; OR $=3.250)$, spiculation $(P=0.002$; OR $=1.949)$, vascular change $P<0.001 ; \mathrm{OR}=2.571)$, and bronchial change $(P=0.036 ; \mathrm{OR}=1.745)$ were independent predictors of increasing the risk of the n-LPA group, whereas small CTR $(P=0.007 ; 0.982)$ was a factor that reduced the risk of the n-LPA group (Table 3 ).

ROC analysis was performed on the mean CT value, CTR, and logistic regression model with significant differences in multivariate analysis, which showed that the AUC was 0.781 [95\% confidence interval (CI) 0.7440.818), 0.593 (95\% CI: 0.547-0.639), and 0.840 (95\% CI: 0.808-0.871), respectively. The optimal cutoff values of the mean CT and CTR were $-472.5 \mathrm{HU}$ (sensitivity, 
Table 3 Ability to distinguish IPA between the LPA group and n-LPA group using the backward elimination process of binary logistic regression

\begin{tabular}{llllll}
\hline Feature & B & SE & $\boldsymbol{P}$ value & OR & $\mathbf{9 5 \% ~ C I}$ \\
\hline Mean CT value & 0.009 & 0.001 & $<0.001$ & 1.009 & $1.007,1.011$ \\
CTR & -0.018 & 0.007 & 0.007 & 0.982 & $0.969,0.995$ \\
Deep lobulation & 1.179 & 0.257 & $<0.001$ & 3.250 & $1.962,5.382$ \\
Spiculation & 0.667 & 0.216 & 0.002 & 1.949 & $1.275,2.977$ \\
Vascular change & 0.944 & 0.231 & $<0.001$ & 2.571 & $1.636,4.041$ \\
Bronchial change & 0.557 & 0.265 & 0.036 & 1.745 & $1.037,2.935$ \\
Constant & 2.346 & 0.660 & $<0.001$ & 10.441 & \\
\hline
\end{tabular}

LPA Lepidic-predominant adenocarcinoma, $n$-LPA Nonlepidic-predominant adenocarcinoma, CTR Consolidation-to-tumor ratio, SE Standard error, OR Odds ratio $(O R>1$ indicates the risk factor of $n-L P A ; O R<1$ indicates the protective factors of $n$-LPA). $P<0.05$ was considered statistically significant

60.5\%; specificity, $83.1 \%$ ) and $27.4 \%$ (sensitivity, 38.4\%; specificity, $83.9 \%)$, respectively. Whereas the sensitivity and specificity of logistic regression model were 76.4 and $78.7 \%$, respectively (Table 4). Logistic regression model conbined with mean CT value, CTR, and morphological HRCT features had significantly higher predictive efficiency than that using only mean $\mathrm{CT}$ value or CTR $(P<0.001)$ (Fig. 5).

\section{Discussion}

To our knowledge, LPA has the best prognosis without lymph node dissection or only lymph node sampling, whereas n-LPA requires systematic lymph node dissection [8], so we performed a retrospective analysis of 630 GGNs that were identified pathologically after resection as early-stage IPA with invasive foci more than $5 \mathrm{~mm}$. Previous studies [11-13] has confirmed that HRCT features had good diagnostic value in differentiating IPA from PIL-MIA, therefore, we excluded PIL and MIA cases and focused on differences in the HRCT features of IPA subtypes. Our previous studies also showed that even with pGGN, 39\% were various subtypes of IPAs [15]. In this cohort, we included pGGNs and mGGNs with CTR $\leq 0.5$, and pGGNs accounted for $65.2 \%$. The number of LPA was greater than that of n-LPA (367/ 263), and the proportion of pGGNs in the LPA group was significantly higher than that in the n-LPA group $(P<0.001)$. This was consistent with the previous report that the ground-glass opacity on HRCT was positively correlated with the lepidic growth pattern of the tumor pathologically [7]. In GGNs, the emergence of various IPA subtypes may be related to the following aspects, APA and PPA appeared as GGN for air-filled sparse hypertrophic areas [13]; This study included three SPAs, which were composed of a mixture of solid-predominant growth pattern and other growth patterns, of which lepidic growth patterns accounts for $40 \%, 30 \%, 30 \%$, and their diameters were all less than $10 \mathrm{~mm}$; MPA is usually manifested as solid nodules, and the micropapillary type in our study was other subtypes containing a small number of micropapillary structures, so it can be manifested as GGN; And beyond that, This discrepancy between radiological and pathological findings could be explained by a partial volume effect, detection of small nonaerated components may be difficult because of inadequate spatial resolution [13]. Although, our all CT images were acquired by using HRCT at $1 \mathrm{~mm}$ or $1.25 \mathrm{~mm}$ slice thickness, a much higher resolution may be needed to detect small nonaerated invasion.

This was the larger study of GGNs for the purpose of differentiating LPA from n-LPA group. We observed that the ratio of IPA was significantly higher in women (65.5\%) and no smokers (79.1\%). The Fleischner Society Guidelines [2] also referred to the incidence of adenocarcinoma in nonsmokers was increasing, with female nonsmokers being affected significantly more often than male nonsmokers. However, the relationship to nodule type was not reported. In our study, there were no significant differences between LPA and n-LPA groups in both sex and smoking history.

In univariate and multivariate analysis showed that deep lobulation, spiculation, vascular change and bronchial change were more common in the n-LPA group than in the LPA group. $(P<0.05)$. Several studies have examined marginal lobulation and spiculation related to "tumor development or invasion" in adenocarcinoma [2, 5, 12]. Zhang et al. [16] showed bronchial change was a predictor of invasiveness. Gao et al. [17] found III and IV vascular changes were more prone to invasive behavior, where type III vessels within lesions are tortuous or rigid without an increase in amount and type IV vessels are more complex vascular changes than the other types,

Table 4 Comparison of the mean CT value, CTR, and ROC curve of the logistic regression model to distinguish IPA between the nLPA and LPA groups

\begin{tabular}{|c|c|c|c|c|c|}
\hline Factor & AUC value (95\% confidence interval) & Cutoff value & Sensitivity (\%) & Specificity (\%) & $P$ \\
\hline Mean CT value & $0.781(0.744-0.818)$ & $-472.5 \mathrm{HU}$ & $60.5 \%$ & $83.1 \%$ & $<0.001$ \\
\hline CTR & $0.593(0.547-0.639)$ & $27.4 \%$ & $38.4 \%$ & $83.9 \%$ & $<0.001$ \\
\hline Logistic model & $0.840(0.808-0.871)$ & 3.958 & $76.4 \%$ & $78.7 \%$ & $<0.001$ \\
\hline
\end{tabular}

Cutoff value: The cutoff value of ROC curve of logistic regression model is the factor value or predictive probability value of the model and the maximum value of Youden's index. LPA Lepidic-predominant adenocarcinoma, $n$-LPA Nonlepidic-predominant adenocarcinoma, CTR Consolidation-to-tumor ratio, AUC Area under the curve. $P<0.05$ was considered statistically significant 


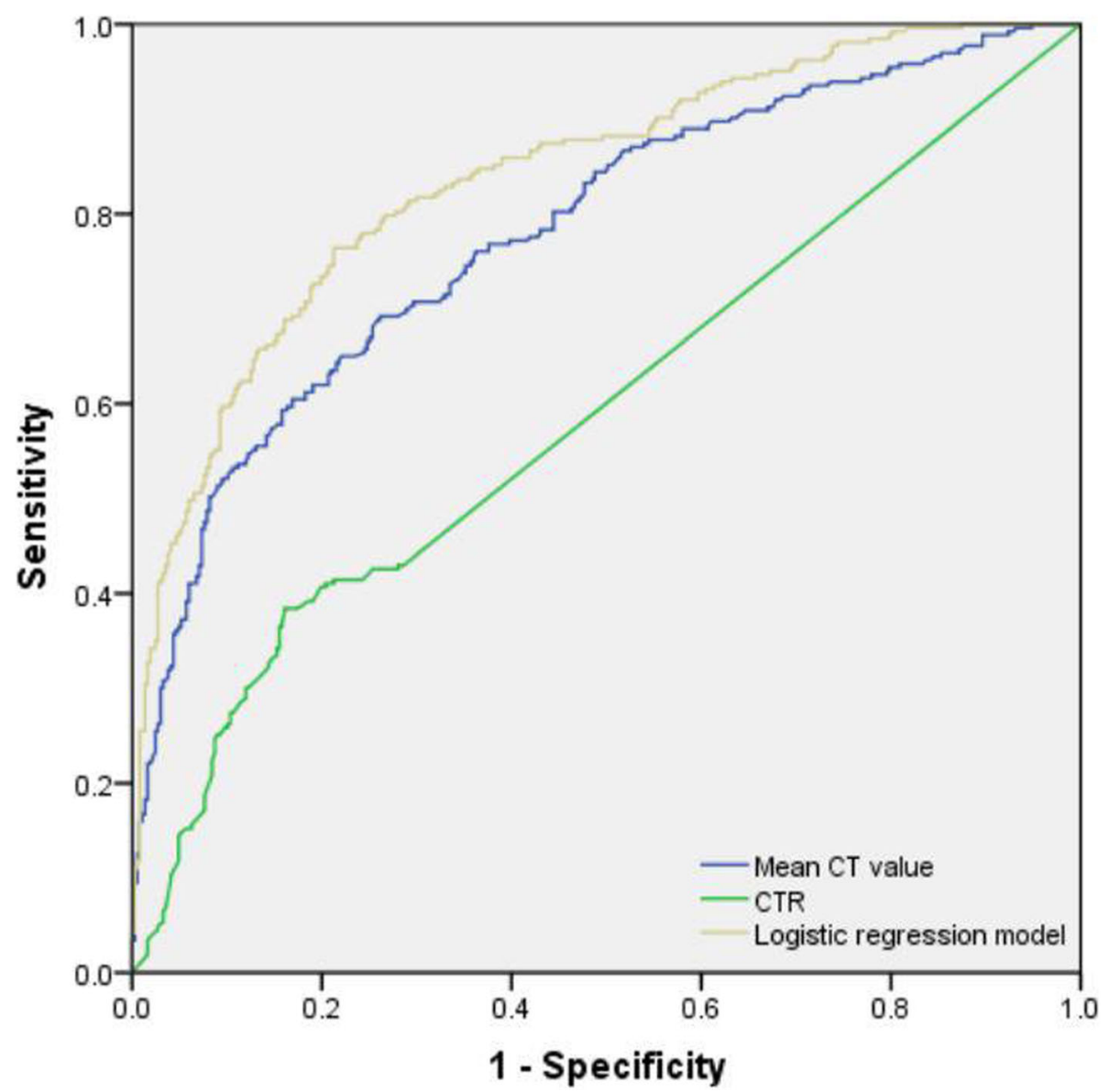

Fig. 5 Ability of the mean $C T$ value, CTR, and logistic regression model to distinguish LPA from n-LPA. Logistic regression model includes mean CT value, CTR, deep lobulation, spiculation, vascular change, and bronchial change. The AUC value $(A \cup C=0.840)$ was significantly higher than the mean $C T$ value ( $A \cup C=0.781$ ) or $C T R(A \cup C=0.593)$, and the diagonal line represents the reference line

such as irregular expansion and convergence of multiple blood vessels. Liang et al. [18] also demonstrated that the number of blood vessels entering GGN (vascular aggregation) was a risk factor for predicting invasiveness. These morphologic features were associated with active fibroblast proliferation in adenocarcinoma and caused by the contraction of fibrous tissues [19]. Noguchi et al. [20] suggested that active fibroblast proliferation in adenocarcinoma was related to the invasive growth of tumors.

Previous studies $[13,14]$ comparing IPA with PILMIA had shown that the CT value of GGN was confirmed to be associated with invasiveness. For example, Zhou et al. [11] showed that the lesion with $\mathrm{CT}$ values in pGGN and mGGN greater than $-583.6 \mathrm{HU}$ and $571.6 \mathrm{HU}$, respectively, was more likely to be IPA. Lee et al. [12] showed that GGNs with CT values of >$472 \mathrm{HU}$ were more likely to be IPAs. This result was similar to the mean CT value $(>-472.5 \mathrm{HU})$ we predicted for the n-LPA, theoretically, however, the mean CT value threshold of IPA subtypes should be greater than that of IPA and MIA-PIL. This may be due to the fact that we used the Freehand ROI but not Circle ROI to draw along the edge of the GGN as large as possible on the maximum transverse axis on the lung window, but Lee used the circular ROI outline causing the loss of the edge part with large gas content for irregular nodules, thus raising the $\mathrm{CT}$ value. However, considering the influence of CT scanning parameters on the CT value of GGN, it was limited to make a diagnosis only by $\mathrm{CT}$ value. In this study, CTR $>27.4 \%$ was more likely to be n-LPA with poor prognosis, which was consistent with the conclusion by Tsutani et al. [21] that showed GGN with CTR $>25 \%$ had a high postoperative recurrence rate. Ko et al. [22] studied 138 cases of stage I lung adenocarcinoma with GGN, and the CTR of LPA and nLPA were 14.5 and $35.4 \%(P=0.002)$, which were higher than the CTR of LPA (13.9\%) and n-LPA (27.8\%) in this study. Considerations were first related to the exclusion of mGGNs with CTR $>0.5$, which reduced the proportion of solid predominant GGNs in the n-LPA group, however, LPA was rare in mGGNs with CTR $>0.5$, so it 
was less affected in LPA, and secondly, the measurement methods of the two studies were inconsistent.

In univariate analysis, there were statistically significant differences in the mean diameter and tumor-lung interface $(P=0.021,<0.001)$, but not in multivariate analysis. Some studies [12, 13] showed the size of the nodule was related to the malignancy of the tumor, however the pathological subtypes of IPA were determined by the proportion of invasive component which was more common in the central region of the GGN [19], while the size of the nodule was determined by the peripheral lepidic growth pattern progressing slowly. In this study, the mean diameter of GGN in the LPA group and the n-LPA group overlapped greatly $(14.49 \pm 5.22 \mathrm{~mm}$ : $15.43 \pm 4.79 \mathrm{~mm})$, so it could not be used as an independent predictor. Hwang et al. [23] also showed that in patients with early pulmonary adenocarcinoma measuring $<3 \mathrm{~cm}$, disease-free survival was remarkably correlated with the size of the solid part of the tumor, but not with the whole tumor. A fuzzy tumor-lung interface was more likely to occur in the LPA group than in the n-LPA group. The lepidic growth pattern part of LPA was more common than that of n-LPA. the lepidic growth pattern was that the tumor cells growing along thickened alveolar walls and air filled in the alveolar cavity [12], when the air content of the peripheral part was close to that of adjacent normal lung tissue and the CT spatial resolution was limited, the tumor-lung interface of GGN on CT was fuzzy. However, the frequency of clear tumor-lung interface was higher in both groups and was not statistically significant in multivariate analysis. No significant differences were observed in pleural indentation sign and bubble-like lucency. Masahiro et al. [24] found that the incidence of pleural indentation increased with increased tumor invasiveness, the volume ratio of solid components in GGN was $>63 \%$ and that the incidence of pleural indentation sign increased. Nevertheless, we investigated GGNs with CTR of $<50 \%$ and did not consider the distance between the nodules and pleura. The incidence of bubble-like lucency in both groups was low, which was related to the observation of multi-directional reconstruction images, excluding the air space connected with the bronchus.

Our study also found that AUC values of the logistic regression model, the mean CT values and CTR were 0.840 (sensitivity, $76.4 \%$, specificity, $78.7 \%$ ), 0.781 (sensitivity, 60.5\%, specificity, 83.1\%), and 0.593 (sensitivity, $38.4 \%$, specificity, $83.9 \%$ ), respectively. The logistic regression model, which obtained by combining deep lobulation, spiculation, vascular change, and bronchial change, the mean CT values and CTR, can improve the sensitivity more than using mean CT value or CTR alone in distinguishing LPA from n-LPA. Differentiating LPA and n-LPA was very important for the preoperative planning of surgical procedures and simultaneously as a reference value for GGN management. For GGN in patients with deep lobulation and vascular and bronchial changes, the follow-up should be terminated and surgical treatment should be considered.

This study has certain limitations. First, this was a retrospective analysis, mainly based on pathological diagnosis. The assessment of IPA subtypes may be inconsistent, especially when multiple subtype components coexisted. Second, this study only focused on lepidic and nonlepidic types of IPA and did not further analyze imaging differences among other pulmonary adenocarcinomas.

\section{Conclusions}

The HRCT features, including deep lobulation, spiculation, vascular change, and bronchial change, may indicate nonlepidic IPA in GGNs, and the diagnosis of nonlepidic IPA is supported when the mean CT value is $>-472.5 \mathrm{HU}$ or CTR is $>27.4 \%$. Simultaneously, the combined application of mean CT value, CTR, and HRCT features can effectively improve the diagnosis of nonlepidic.

\section{Abbreviations \\ PLA: People's Liberation Army; HRCT: High-resolution computed tomography; IPA: Invasive pulmonary adenocarcinoma; GGN: Ground-glass nodule; pGGN: Pure GGN; mGGN: Mixed GGN; CTR: Consolidation-to-tumor ratio; WHO: World Health Organization; PIL: Preinvasive lesions; MIA: Minimally invasive adenocarcinoma; LPA: Lepidic-predominant adenocarcinoma; APA: Acinar-predominant adenocarcinoma; PPA: Papillary-predominant adenocarcinoma; MPA: Micropapillary-predominant adenocarcinoma; SPA: Solid-predominant adenocarcinoma; n-LPA: Nonlepidic-predominant adenocarcinoma; iCT: Information and Communication technology; GE: General Electric Company; IBM: International Business Machine; WI: Wisconsin; HU: Hounsfield unit; KV: Kilovolt; WL: window level; WD: Window width; HE: Hematoxylin-eosin; ROC: Receiver operating characteristic; AUC: Area under the curve; OR: Odds ratio; Cl: Confidence interval; SE: Standard error; M: Median; Q1: Quartile1; Q3: Quartile3; vs: Versus; RUL: Right upper lobe; RML: Right middle lobe; RLL: Right lower lobe; LUL: Left upper lobe; LLL: Left lower lobe; R\&D: Research and development}

\section{Acknowledgements}

The pathologist of Gao Jie has spent a lot of time on the pathological reconfirmation of our included cases, and here we would like to express our sincere gratitude to her.

\section{Authors' contributions}

PZ and TL: Conceptualization, formal analysis, visualization, writing original draft, and editing. SZ: Conceptualization, supervision, project administration, and review. XT and XJ: Data curation, investigation, review and editing. SZ had full access to all the data in the study and take responsibility for the integrity of the data and the accuracy of the data analysis. All authors have read and approved the final version of manuscript.

\section{Funding}

This study was supported by the National Key R\&D Program of China (2017YFC1308700) and the National Natural Science Foundation of China (81641068)

\section{Availability of data and materials}

The dataset used and/or analyzed during the current study are available from corresponding author on reasonable request. 


\section{Declarations}

\section{Ethics approval and consent to participate}

The study was approved by the Ethics Committee of Fourth Medical Center of Chinese PLA General Hospital (approval No. 2019YL002-HS001). Written informed consent was obtained from all study participants.

\section{Consent for publication}

Not applicable.

\section{Competing interests}

The authors have declared that they have no conflicts of interests.

\section{Author details}

'Department of Radiology, Fourth Medical Center of PLA General Hospital, 51 Fucheng Road, Beijing 100048, China. '2Department of Radiology, First Medical Center of PLA General Hospital, 28 Fuxing Road, Beijing 100853, China.

Received: 25 December 2020 Accepted: 1 October 2021

Published online: 19 October 2021

\section{References}

1. Stang A, Schuler M, Kowall B, Darwiche K, Kühl H, Jöckel KH. Lung Cancer screening using low dose CT scanning in Germany. Extrapolation of results from the National Lung Screening Trial. Dtsch Arztebl Int. 2015;112(38):63744. https://doi.org/10.3238/arztebl.2015.0637.

2. MacMahon H, Naidich DP, Goo JM, Lee KS, Leung ANC, Mayo JR, et al. Guidelines for Management of Incidental Pulmonary Nodules Detected on CT images: from the Fleischner society 2017. Radiology. 2017;284(1):228-43. https://doi.org/10.1148/radiol.2017161659.

3. Kim HY, Shim YM, Lee KS, Han J, Yi CA, Kim YK. Persistent pulmonary nodular ground-glass opacity at thin-section CT: histopathologic comparisons. Radiology. 2007;245(1):267-75. https://doi.org/10.1148/ra diol.2451061682.

4. Matsunaga T, Suzuki K, Takamochi K, Oh S. What is the radiological definition of part-solid tumour in lung cancer? Eur J Cardiothorac Surg. 2017;51(2):242-7. https://doi.org/10.1093/ejcts/ezw344.

5. Travis WD, Brambilla E, Nicholson AG, Yatabe Y, Austin JHM, Beasley MB, et al. The 2015 World Health Organization classification of lung tumors: impact of genetic, clinical and radiologic advances since the 2004 classification. J Thorac Oncol. 2015;10(9):1243-60. https://doi.org/10.1097/ JTO.0000000000000630.

6. Mansuet LA, Bobbio A, Blons $\mathrm{H}$, et al. The new histologic classification of lung primary adenocarcinoma subtypes is a reliable prognostic marker and identifies tumors with different mutation status: the experience of a French cohor. Chest. 2014;146(3):633-43. https://doi.org/10.1378/chest.13-2499.

7. Travis WD, Brambilla E, Noguchi M, Nicholson AG, Geisinger KR, Yatabe Y, et al. International association for the study of lung cancer/American thoracic society/European respiratory society international multidisciplinary classification of lung adenocarcinoma. J Thorac Oncol. 2011;6(2):244-85. https://doi.org/10.1097/JTO.0b013e318206a221.

8. Cheng X, Zheng D, Li Y, Li H, Sun Y, Xiang J, et al. Tumor histology predicts mediastinal nodal status and may be used to guide limited lymphadenectomy in patients with clinical stage I nonsmall cell lung cancer. Thorac Cardiovasc Surg. 2018;155(6):2648-56. https://doi.org/10.101 6/j.jtcvs.2018.02.010.

9. Jiang G, Chen C, Zhu Y, et al. Shanghai Pulmonary Hospital Experts Consensus on the management of ground-glass nodules suspected as lung adenocarcinoma (Version 1). Zhongguo Fei Ai Za Zhi. 2018;21(3):147-59. https://doi.org/10.3779/j.issn.1009-3419.2018.03.05.

10. Liu S, Wang R, Zhang Y, Li Y, Cheng C, Pan Y, et al. Precise diagnosis of intraoperative frozen section is an effective method to guide resection strategy for peripheral small-sized lung adenocarcinoma. J Clin Oncol. 2016; 34(4):307-20. https://doi.org/10.1200/JCO.2015.63.4907.

11. Zhou QJ, Zheng ZC, Zhu YQ, Lu PJ, Huang J, Ye JD, et al. Tumor invasiveness defined by IASLC/ATS/ERS classification of ground-glass nodules can be predicted by quantitative CT parameters. Thorac Dis. 2017; 9(5):1190-200. https://doi.org/10.21037/jtd.2017.03.170.

12. Lee HY, Choi YL, Lee KS, Han J, Zo Jl, Shim YM, et al. Pure ground-glass opacity neoplastic lung nodules: histopathology, imaging, and management. AJR Am J Roentgenol. 2014;202(3):W224-33. https://doi.org/1 0.2214/AJR.13.11819.

13. Eguchi T, Yoshizawa A, Kawakami S, Kumeda H, Umesaki T, Agatsuma H, et al. Tumor size and computed tomography attenuation of pulmonary pure ground-glass nodules are useful for predicting pathological invasiveness. PLoS One. 2014;9(5):e97867. https://doi.org/10.1371/journal. pone.0097867.

14. Yoshizawa A, Motoi N, Riely GJ, Sima CS, Gerald WL, Kris MG, et al. Impact of proposed IASLC/ATS/ERS classification of lung adenocarcinoma: prognostic subgroups and implications for further revision of staging based on analysis of 514 stage I cases. Mod Pathol. 2011;24(5):653-64. https://doi.org/10.1038/ modpathol.2010.232.

15. Jin X, Zhao SH, Gao J, Wang DJ, Wu J, Wu CC, et al. CT characteristics and pathological implications of early stage (T1NOM0) lung adenocarcinoma with pure ground-glass opacity. Eur Radiol. 2016;25(9):2532-40. https://doi. org/10.1007/s00330-015-3637-Z.

16. Zhang Y, Qiang JW, Shen Y, Ye JD, Zhang J, Zhu L. Using air bronchograms on multi-detector $C T$ to predict the invasiveness of small lung adenocarcinoma. Eur J Radiol. 2016;85(3):571-7. https://doi.org/10.1016/j.ejra d.2015.12.014

17. Gao F, Li M, Ge X, Zheng X, Ren Q, Chen Y, et al. Multi-detector spiral CT study of the relationships between pulmonary ground-glass nodules and blood vessels. Eur Radiol. 2013;23(12):3271-7. https://doi.org/10.1007/s0033 0-013-2954-3.

18. Liang J, Xu XQ, Xu H, Yuan M, Zhang W, Shi ZF, et al. Using the CT features to differentiate invasive pulmonary adenocarcinoma from pre-invasive lesion appearing as pure or mixed ground-glass nodules. Br J Radiol. 2015; 88(1053):20140811. https://doi.org/10.1259/bjr.20140811.

19. Sakurai H, Maeshima A, Watanabe S, Suzuki K, Tsuchiya R, Maeshima AM, et al. Grade of stromal invasion in small adenocarcinoma of the lung histopathological minimal invasion and prognosis. Am J Surg Pathol. 2004; 28(2):198-206. https://doi.org/10.1097/00000478-200402000-00007.

20. Noguchi M, Morikawa A, Kawasaki M, Matsuno Y, Yamada T, Hirohashi S, et al. Small adenocarcinoma of the lung: histologic characteristics and prognosis. Cancer. 1995;75(12):2844-52. https://doi.org/10.1002/1097-0142 (19950615)75:12<2844::AID-CNCR2820751209>3.0.CO;2-\#.

21. Tsutani $Y$, Miyata $Y$, Nakayama H, Okumura S, Adachi S, Yoshimura M, et al. Oncologic outcomes of segmentectomy compared with lobectomy for clinical stage IA lung adenocarcinoma: propensity score-matched analysis in a multicenter study. J Thorac Cardiovasc Surg. 2013;146(2):358-64. https:// doi.org/10.1016/j.jtcvs.2013.02.008.

22. Ko JP, Suh J, Ibidapo O, Escalon JG, Li J, Pass H, et al. Lung adenocarcinoma: correlation of quantitative $C T$ findings with pathologic findings. Radiology 2016;280(3):931-9. https://doi.org/10.1148/radiol.2016142975.

23. Hwang EJ, Park CM, Ryu Y, Lee SM, Kim YT, Kim YW, et al. Pulmonary adenocarcinomas appearing as part-solid ground-glass nodules: is measuring solid component size a better prognostic indicator? Eur Radiol. 2015:25(2):558-67. https://doi.org/10.1007/s00330-014-3441-1.

24. Yanagawa M, Tanaka Y, Leung AN, Morii E, Kusumoto M, Watanabe S, et al. Prognostic importance of volumetric measurements in stage I lung adenocarcinoma. Radiology. 2014;272(2):557-67. https://doi.org/10.1148/ra diol.14131903

\section{Publisher's Note}

Springer Nature remains neutral with regard to jurisdictional claims in published maps and institutional affiliations.

Ready to submit your research? Choose BMC and benefit from:

- fast, convenient online submission

- thorough peer review by experienced researchers in your field

- rapid publication on acceptance

- support for research data, including large and complex data types

- gold Open Access which fosters wider collaboration and increased citations

- maximum visibility for your research: over $100 \mathrm{M}$ website views per year

At BMC, research is always in progress.

Learn more biomedcentral.com/submission 